\title{
Ciencia, Tecnología, Sociedad e Innovación en la Sociedad del Conocimiento: a ambos lados de las «divisorias del aprendizaje»
}

\author{
AMALIA VIJANDE MARTÍNEZ \\ Universidad de Oviedo
}

RESUMEN. En nuestras sociedades crecientemente dependientes de la tecnociencia, reflexionar sobre las relaciones entre ciencia, tecnología, sociedad e innovación $(\mathrm{CTS}+\mathrm{I})$ se ha convertido en una tarea inexcusable. El campo interdisciplinar de estudios sobre CTS + I, que se había venido consolidado desde los años sesenta y setenta en los países occidentales, ha vivido en los últimos años un impulso muy importante en el ámbito iberoamericano. Fruto de este creciente interés es la colección de libros CTS + I editados por Cambridge University Press en colaboración con la OEI (Organización de Estados Iberoamericanos para la Educación, la Ciencia y la Cultura). En esta nota se reseñan los cinco primeros títulos de la citada colección.

La ciencia y la tecnología, a partir sobre todo de las últimas décadas del siglo $\mathrm{xx}$, se han convertido en factores absolutamente determinantes de la vida social. Estamos rodeados de ciencia y tecnología por todas partes; desde los impresionantes artefactos destinados a la investigación espacial, quizás el prototipo de gran empresa científico-tecnológica que los ciudadanos corrientes no vemos más que en la televisión o el cine, hasta el más
ABSTRACT. The analysis of the relationships amog science, technology, society and innovation (STS + I) has become an inevitable task in contemporary societies, increasingly dependent on technoscience. The interdisciplinary studies on STS + I, which crystalized in Western societies during the last four decades, have experimented recently an important development in Latin America. An example of this increasing interest is the book series on STS + I published by Cambridge University Press in association with OEI (Organización de Estados Iberoamericanos para la Educación, la Ciencia y la Cultura). In this article the first five volumes of this series are reviewed.

pequeño rincón de nuestra casa o el aspecto más íntimo de nuestra vida privada, que es posible que ya no seamos capaces de concebir sin la mediación de la ciencia y la tecnología. Pero además de la proliferación de artefactos, nuestra forma de comunicarnos, interaccionar con los demás, trabajar o disfrutar del ocio, todas nuestras relaciones sociales en definitiva, están igualmente mediadas por la ciencia y la tecnología.

* Este trabajo ha sido posible gracias a la financiación del Ministerio de Ciencia y Tecnología a través del proyecto BFF02 01102. 
Pero tal vez esta imagen de la ciencia y la tecnología sólo sea posible en un determinado sector del planeta. Seguro que, mirando un poco más allá de nuestras casas, sí podemos concebir lugares donde no es tan fácil, antes al contrario, encontrar ciencia y tecnología en la vida de la gente. $\mathrm{Ni}$ siquiera la más básica y elemental, como la tecnología médica o aquella destinada a mejorar la habitabilidad de las viviendas. De hecho, parece que cuanto más se «universalizan» la ciencia y la tecnología en «nuestro lado» del mundo, más se dificulta su introducción en «el otro lado».

Mirando de momento sólo a nuestro «mundo desarrollado», la proliferación de la ciencia y la tecnología (o, también, utilizando el término de moda, de la tecnociencia -Echeverría, 1999-), a partir de la segunda mitad del siglo Xx aproximadamente, coincidió con el nacimiento de actitudes de rechazo y temor ante ellas en la sociedad en general, tanto respecto a sus posibles riesgos para la vida humana y la salud del planeta (Carson, 1962) como en lo referente a los cambios que suponía la invasión (y la consiguiente transformación) científico-tecnológica de todos los ámbitos de las relaciones sociales (Roszak, 1968). En el ámbito académico, esta sensación se materializó en la aparición (que podemos fechar a finales de los sesenta y principios de los setenta) de estudios de la ciencia y la tecnología, y su relación con la sociedad, tanto en los momentos previos de concepción y desarrollo de investigaciones y artefactos como en su aplicación y consecuencias. Los estudios sobre Ciencia, Tecnología y Sociedad (CTS) surgieron en países de habla inglesa (Reino Unido y Estados Unidos específicamente), con carácter crítico e interdisciplinar (desde disciplinas como la historia de la ciencia, la filosofía de la ciencia y la tecnología, la sociología del conocimiento o la economía del cambio técnico), y actualmente se han constituido como uno de los campos de trabajo en el estudio de la ciencia y la tecnología más prolíficos en el panorama internacional (para un recorrido por la historia de los estudios CTS, véase González García, M., et al., 1996). Uno de sus principales características era (y es) la consideración de la ciencia y la tecnología no como fenómenos regidos por una lógica interna, construidos mediante la aplicación de un estricto método cognitivo, y valorativamente neutrales, sino como realidades producto del contexto social, cultural, económico e histórico en el que surgen, y que a su vez tienen profundas consecuencias sociales, culturales y económicas.

En la región iberoamericana, los estudios CTS están alcanzando una importante presencia en el ámbito de la investigación y la docencia de la ciencia y la tecnología. Y cuando estos estudios se llevan a cabo desde una perspectiva diferente, periférica (desde «el otro lado» que decíamos antes), las preocupaciones que vertebran la investigación cambian, se trasladan, y otros problemas entran en juego. Por ejemplo, la ausencia de tecnología, pasa a ser el problema, y no su proliferación exagerada. La cuestión de la distribución equitativa de riesgos tecnológicos y consecuencias negativas de la tecnociencia debe conjugarse con la necesidad de crear riqueza mediante innovación tecnológica para mejorar las condiciones de vida de los ciudadanos y favorecer el crecimiento económico.

En conclusión, los estudios CTS hechos desde la periferia complementan los análisis construidos desde el Norte desarrollado, introduciendo enfoques enriquecedores y nuevos temas de investigación. La Organización de Estados Iberoamericanos para la Educación, la Ciencia y la Cultura (OEI), entidad intergubernamental de cooperación internacional, inició en 1999 un programa dentro de su área de cooperación científica destinado a promover el enfoque Ciencia, Tecnología, Sociedad e Innovación (CTS + I), en el que el estudio de los aspectos sociales y éticos de la cien- 
cia y la tecnología se conjuga con la preocupación por la innovación científico-tecnológica, es decir, por el papel de la ciencia y la tecnología en el desarrollo económico y social. Una de las acciones principales dentro de este programa ha sido la edición, junto con Cambridge University Press, de una colección de temática CTS + I, que hasta la fecha cuenta con cuatro títulos, a los que en breve se sumará un quinto. En ellos se puede encontrar una panorámica muy amplia de las cuestiones y problemas de este campo de estudio, aunque, como corresponde a una colección en desarrollo, esta panorámica no sea completa: el análisis de un caso concreto de desarrollo tecnológico y de su contexto social y ambiental (la política forestal en el Principado de Asturias, en Políticas del bosque, de José A. López Cerezo y M. I. González García, 2002), el estudio más general de una rama de la tecnociencia, la biotecnología (en Biotecnología y sociedad, Emilio Muñoz, 2002), la reflexión sobre la problemática de la innovación vista desde la periferia (Navegando contra el viento, Ciencia, Tecnología y Subdesarrollo, Rodrigo Arocena y Judith Sutz, de próxima aparición), la visión desde la educación de los problemas que afectan a la humanidad y el planeta de forma global (Construyamos un futuro sostenible, Amparo Vilches y Daniel Gil, 2003) y el papel de la institución universitaria en el contexto académico y social contemporáneo [La universidad (im)posible, Jacques Marcovitch, 2002].

Una de las metodologías ya tradicionales de los estudios CTS es mostrar la dinámica de las relaciones entre Ciencia, Tecnología y Sociedad mediante el análisis exhaustivo de casos concretos de desarrollo tecnológico. Este método ha sido ampliamente aplicado por autores europeos (por ejemplo, en Bijker, W.; Hughes, T., y Pinch, T., 1987), pero todavía no ha encontrado un lugar importante en la literatura CTS en castellano. Políticas del bosque asume esta metodología, y toma como punto de partida la política forestal en Asturias y, en concreto, el «problema del eucalipto», durante los últimos veinte años aproximadamente, para realizar una reflexión más amplia sobre la necesidad de la participación pública en la implantación y evaluación de tecnologías, y, en particular, en las acciones de intervención ambiental.

La conclusión que los autores extraen del repaso exhaustivo de toda la polémica sobre el problema del eucalipto (cuyo punto álgido fue la «revuelta» de los habitantes de un pueblo costero asturiano contra la plantación de eucaliptos en los terrenos próximos al pueblo en 1988) es que no sólo el debate público, sino también las consecuencias indeseadas de la gestión forestal en Asturias (de carácter ecológico, económico y social) fueron causados por la reducción a sus aspectos técnicos de un problema que era desde el principio social y complejo. Ya desde el comienzo de la controversia saltaron a las páginas de los periódicos una legión de expertos (ingenieros, biólogos...) que, desde las filas de los opositores a los eucaliptos o desde las de sus defensores, recurrían a la ciencia y el conocimiento técnico para justificar sus posturas. Posteriormente, la Administración elaboró una legislación que, pese a presentarse como sensible a las preocupaciones de la opinión pública, cayó igualmente en una tecnificación del problema, reduciéndolo sólo a una cuestión dependiente de la elaboración de informes técnicos en los que la opinión no experta no tenía ningún papel.

López Cerezo y González García consideran, siguiendo a investigadores como Funtowicz y Ravetz, que la tecnociencia contemporánea se ha convertido, en buena medida, en una empresa caracterizada por la necesidad de tomar decisiones políticas urgentes sobre problemas muy complejos, que involucran parámetros sociales, culturales, políticos, económicos y ambientales 
muy diversos, relacionados en un equilibrio muy delicado. Es lo que denominan «ciencia postnormal» (Funtowicz, S., y Ravetz, J., 1993). En estos contextos complejos («sociosistemas»), la ciencia se ve obligada, si quiere obtener resultados manejables políticamente, a reducir las innumerables variables a unas pocas, lo que hace que sus resultados estén marcados por la incertidumbre. Y los factores que se reducen son siempre aquellos relacionados con el conocimiento no experto, o que no son fácilmente cuantificables, reducibles a números (como las cuestiones valorativas, estéticas...). Ésta es la conclusión que los autores obtienen del caso de estudio: el eucalipto se introduce en un sociosistema complejo, afectando así al equilibrio entre muchos factores ecológicos, económicos y sociales; y el problema se «soluciona» políticamente tomando unos pocos de esos factores y reduciendo el problema para que sea técnicamente manejable, omitiendo en todo el proceso otras fuentes de información de carácter popular o profano. El resultado: una gestión inadecuada que no obtiene soluciones sostenibles.

El trabajo utiliza el caso de estudio para extraer conclusiones generales para la concepción de la tecnología y para su evaluación, y para reclamar un nuevo modelo de participación pública en la evaluación y gestión de tecnologías. La evaluación de tecnologías clásica (Jones, M. V., 1971), de la que las evaluaciones de impacto ambiental son una variedad muy significativa, se basa en una concepción de la tecnología como «un cuerpo de conocimiento especializado que deriva de la aplicación de la ciencia y tiene como objetivo el diseño y desarrollo de artefactos» (Políticas del bosque, p. 97). Se plantea como un análisis de impactos y riesgos sociales, que permita minimizar las consecuencias negativas de las tecnologías y evitar su rechazo por parte de la opinión pública. De modo que la evaluación de tecnologías se configura como una acción reactiva ante sus impactos. Los autores, por su parte, defienden un modelo constructivo de evaluación, que parta de una concepción de la tecnología como una forma de organización social, y que se plantee como una forma de influir activamente en el desarrollo e implantación de las tecnologías, no sólo por parte de los expertos, sino también de los ciudadanos legos afectados o interesados. El objetivo principal es anticipar impactos, no reaccionar ante ellos, y además, anticipar todos los impactos que se puedan prever para cualquier grupo social implicado, directa o indirectamente, en el desarrollo o la implantación de una determinada innovación. Por esto resulta fundamental promover la participación de todos los actores sociales en estos procesos de toma de decisiones en política científico-tecnológica y ambiental.

$\mathrm{Si}$ las intervenciones medioambientales pueden ser consideradas como un prototipo de desarrollo tecnológico contemporáneo, la biotecnología puede considerarse como un prototipo de la nueva tecnociencia. Además, la biotecnología es un caso claro de vinculación con la sociedad, porque sus avances, desde la clonación a los nuevos medicamentos, son seguidos muy de cerca por la opinión pública, llenan a diario en las páginas de los periódicos, mucho más que otras disciplinas científicas que resultan más lejanas para la ciudadanía, como la física o la química. Por esto la biotecnología ofrece un campo de estudio adecuado para estudiar las interacciones entre ciencia y tecnología, por un lado, entre tecnociencia y sociedad, por otro, y entre desarrollo tecnocientífico e innovación y desarrollo empresarial. Emilio Muñoz es un investigador en bioquímica y biología molecular, que ha tenido un papel muy activo durante años en la política científica española y europea, y, en los últimos tiempos, ha dedicado su trabajo al análisis de las relaciones entre Ciencia, Tecnología y Sociedad. El currículo de este autor permite, en primer lugar, adivinar el carácter multidisciplinar 
de los estudios CTS y, en segundo, ofrece las garantías de que su estudio Biotecnología y sociedad no sólo ofrecerá la reflexión crítica del estudioso CTS, sino también la visión del científico que conoce el campo de primera mano, y del gestor responsable de tareas legislativas y regulativas.

Biotecnología y sociedad analiza el conocimiento científico y técnico que está en la base de las biotecnologías, realizando un recorrido histórico desde sus orígenes en sus antepasados tradicionales (como las prácticas de modificación de alimentos por fermentación, por ejemplo, o las prácticas tradicionales de mejora de especies de animales en agricultura o ganadería), pasando por el nacimiento de la biotecnología científico-técnica en el siglo XIX, y siguiéndoles la pista hasta los últimos y más polémicos desarrollos de la investigación punta (animales y plantas transgénicos, bioinformática, clonación de animales...). Su panorámica de las tres grandes ramas de la biotecnología (agroalimentación, biotecnología ambiental, y medicina y farmacología) dibuja un cuadro completo del repertorio de técnicas, instrumentos y tecnologías biotecnológicas, así como de las investigaciones científicas que hay detrás, de sus aplicaciones comerciales y de las empresas que las desarrollan y comercializan. Para completar su repaso por el panorama de la biotecnología contemporánea, realiza un resumen muy completo de las coyunturas nacionales de los principales países productores de biotecnología, con cuidado en el surgimiento, desarrollo y estructura de las empresas biotecnológicas, y de la legislación y normativa específica en cada caso, así como de las reacciones de la sociedad ante las distintas tecnologías. Por ello esta obra constituye un documento esencial para conocer todos los aspectos de una de las tecnologías que están alcanzando mayores desarrollos actualmente, y cuyos avances constituyen uno de los temas científicotecnológicos que más ocupan y preocupan a la opinión pública.
El estudio de Muñoz, consciente de la complejidad del tema, emplea un enfoque integrador de la biotecnología en su conjunto, aun dentro de la compleja estructura de contextos nacionales, sectores productivos y disciplinas científicas, y también una visión particularizada sobre casos de estudio concretos, como la agricultura biotecnológica (organismos modificados genéticamente) o la terapia génica. Siguiendo esta metodología, dedica una segunda parte de su libro a estudiar las repercusiones socio-económicas de las biotecnologías, y su interacción con el diálogo político y social.

La reacción social ante la biotecnología es generalmente positiva en lo referente a avances médicos o farmacológicos concretos, como la producción de insulina sintética o las actuaciones medioambientales, pero se vuelve crítica feroz y rechazo cuando se trata de actuaciones agroalimentarias (como muestra el rechazo casi total de la opinión pública europea ante los alimentos transgénicos). Muñoz encuentra tres motivos a este rechazo: la ausencia de beneficios claros, la implicación de grandes empresas multinacionales que, se cree, se enriquecen a costa de los pequeños productores, y la novedad de las técnicas, que genera desconfianza. Los alimentos transgénicos son una muestra de la conflictiva relación entre tecnociencia, industria y sociedad que generan las nuevas biotecnologías, una de cuyas principales causas es la toma de conciencia colectiva de que la sociedad actual es, usando el término de Beck, una «sociedad del riesgo» (Beck, U., 1986). De nuevo nos encontramos con la cuestión de la evaluación de riesgos, tema clave en los estudios CTS: la sociedad reclama la evaluación de estos riesgos, no completamente controlables, y un cambio de actitud en su gestión: de una actitud consecuencialista, que se preocupa por solucionar, mediante acciones reactivas de tipo tecnológico o judicial, los problemas causados por la tecno- 
logía, a una actitud preventiva, que mediante el estudio de casos y la regulación adecuada, permita limitar los riesgos antes de que éstos se concreten (López Cerezo, J. A., y Luján, J. L. 2000). En cualquier caso, reclama Muñoz, la solución a las tensiones pasa por hacer la información sobre las biotecnologías, sus beneficios y riesgos, accesible a todos los ciudadanos.

La idea general que subyace a los análisis tradicionales de percepción pública de la tecnología es que la percepción negativa se corresponde siempre con un déficit de conocimiento: la gente que rechaza una tecnología o una línea de investigación científica lo hace porque no está suficientemente bien informada sobre ella. Esta idea tiene detrás la visión de la ciencia y la tecnología como moralmente neutrales y esencialmente benefactoras de la humanidad. Sin embargo, Muñoz rechaza esta idea y señala que los aspectos culturales y los valores de la población en la que se insertan es fundamental para comprender la reacción ante las tecnologías. De hecho, se comprueba con estudios como los eurobarómetros que, generalmente, la gente con una percepción más negativa de las innovaciones tecnocientíficas es aquella más científicamente ilustrada, y la situación en Europa varía considerablemente dependiendo del contexto: por ejemplo, los países productores de biotecnologías son mayoritariamente partidarios de su uso, mientras que la actitud es la contraria en los países consumidores. También es distinta la imagen de la ciencia básica relacionada con la biotecnología (generalmente positiva) de la imagen de las aplicaciones prácticas de la misma.

El repaso a las regulaciones legislativas de las biotecnologías, tanto en Estados Unidos como en Europa, pone de manifiesto una tensión fundamental entre dos exigencias igualmente irrenunciables: por un lado, deben tener presentes las exigencias de la economía de mercado, por ejemplo en lo referente a patentes, y por otro, deben satisfacer las demandas referentes a los derechos individuales y la sostenibilidad del desarrollo. La solución, defiende Muñoz, pasa por asumir una perspectiva contextualista o consecuencialista que tenga en cuenta la justicia social y las consecuencias de los procesos biotecnológicos, así como la participación del mayor número de actores implicados en la toma de decisiones. El principio que debe guiar toda regulación es el principio de precaución o prudencia, que considere al riesgo de forma previa a la actuación, y no posteriormente, como una consecuencia inevitable a abordar una vez que los problemas se producen.

La biotecnología es uno de los campos donde se suscitan los debates públicos más enconados entre la necesidad de innovación tecnológica y de fomentar un desarrollo sostenible, ya que sus consecuencias, actuales o previsibles, pueden resultar de una importancia fundamental para la salud medioambiental del planeta. Por ejemplo, los organismos transgénicos son uno de los resultados tecnológicos más cuestionados y rechazados por la opinión pública, por los supuestos riesgos de amenaza a la biodiversidad que supondría su diseminación en el medio ambiente. También se plantean problemas relacionados con el esquema de distribución de la riqueza que supone el dominio en el mercado internacional de semillas y alimentos de las multinacionales agroalimentarias. Amparo Vilches y Daniel Gil han recogido este y otros muchos temas relacionados con la salud, medioambiental y social, del planeta, en Construyamos un futuro sostenible. Parten de una llamada de atención urgente: el mundo está atravesando una situación de «emergencia planetaria», según atestiguan numerosos investigadores del ámbito académico y organizaciones políticas supranacionales (como la Comisión Mundial del Medio Ambiente y el Desarrollo, o las conferencias de las Naciones Unidas). En concreto, tienen muy en cuen- 
ta los movimientos de organizaciones como la ONU, que han ido estudiando el estado de la cuestión a nivel mundial desde los sesenta, en diversas cumbres y reuniones (como la Cumbre de la Tierra de Río de 1992). Todos coinciden en que el crecimiento y expansión industrial, el agotamiento de recursos... ha llegado a límites difícilmente soportables a nivel humano y ambiental, casi irreversibles. La obra pretende llevar a cabo un planteamiento global de los problemas, que explore todas sus dimensiones y que, además de estas voces de expertos, recoja también las de ciudadanos, docentes y educadores, políticos y gestores, y científicos. El tono de la reflexión es optimista y orientado a la acción, sin caer en el derrotismo que parecería la conclusión obvia de la exposición sistemática de los gravísimos problemas que afectan al ambiente y la humanidad.

Además de lo ambicioso de su tema, el libro de Vilches y Gil destaca por su forma: la narración se realiza en forma de diálogo entre dos personajes, que además plantean sus reflexiones como una invitación al lector para unirse a la conversación. Pero la forma aparentemente descuidada del texto desvela una estructura clara en cuanto comienza la lectura, que se compone de tres grandes bloques: el primero plantea, de forma concreta y minuciosa, los problemas que afectan a la humanidad y al planeta; el segundo analiza las causas de estos problemas, y el tercero propone una reflexión sobre soluciones y alternativas.

Los problemas que los autores analizan son la contaminación ambiental, la degradación de las ciudades, el agotamiento de los recursos, la degradación de los ecosistemas que amenaza a la biodiversidad, y la pérdida de la diversidad cultural. De todos ellos hacen un repaso completo y detallado, que da cuenta tanto de los problemas fácilmente detectados por todos y que forman parte de las preocupaciones ciudadanas habituales como de otros, menos conocidos para los no expertos, pero igualmente importantes. Resulta especialmente interesante su dedicación a problemas que aparentemente no están relacionados con el desarrollo científico y tecnológico, como la degradación del entorno humano, que también resultan fundamentales si deseamos conocer la situación real del planeta, incluyendo a las sociedades que lo habitan. De este modo su recorrido resulta especialmente completo y profundo.

La segunda parte de la obra se fija en el estudio de las causas de los problemas. La ideología tradicional que acompañaba al desarrollo científico-tecnológico, y que aún ahora aparece con frecuencia, identificaba desarrollo científico con desarrollo tecnológico, y a ambos con desarrollo social, en un mecanismo que se creía inevitable y beneficioso. Ésta es la ideología que ha justificado el crecimiento desmesurado que, en gran parte, es la causa de que la situación del mundo haya alcanzado tal grado de insostenibilidad. Nada que pudiera hacer la ciencia (neutral o incluso intrínsecamente beneficiosa) podía ser sino un beneficio social a corto o largo plazo. La sociedad en la que vivimos es una sociedad del riesgo, pero ese riesgo debe ser aceptado tal cual viene, a cambio de un beneficio mayor. Los autores reconocen que el papel de la ciencia y la tecnología, y el de esta ideología del progreso que las acompañaba (Sarewitz, D., 1996), es una de las causas del problema global, pero no la única: defienden un planteamiento holístico, no reduccionista, que considere todos los aspectos de los problemas: físicos, sociales, políticos, económicos, culturales y científico-técnicos. Lejos de buscar una causa aislada para cada problema concreto, ensayan una explicación plural y compleja. Así, por ejemplo, una de las causas de los problemas de sostenibilidad que analizan, además de la expansión de la ciencia y la tecnología, es el hiperconsumo de las sociedades occidentales, o la explosión demográfica, o las economías que buscan sólo beneficios a corto plazo. 
Por último, en una tercera parte se ocupan de proponer caminos que permitan solucionar esta situación de emergencia planetaria tal como la han definido. El tono general de la obra es de un moderado optimismo sobre la posibilidad de transitar caminos alternativos y, partiendo de análisis fiables de las situaciones, pretende llegar a una comprensión global de los problemas, buscando conjugar la conservación del medio ambiente con el desarrollo, e implicar a todos los sectores productivos, desde la agricultura a la industria, y a todos los países de forma global.

Como hemos dicho, no parten del supuesto de que las causas sean únicamente científico-tecnológicas, por lo que no se limitan en sus propuestas a postular salidas tecnológicas que arreglen lo que la tecnología ha estropeado. Pero sí consideran necesaria una reorientación de la ciencia y la tecnología, en la que la innovación tecnológica esté políticamente orientada al desarrollo sostenible, promoviendo tanto criterios universales (como la renovabilidad de los recursos, la búsqueda de la máxima eficiencia y la asimilabilidad de los residuos) con pautas locales que adapten la innovación al contexto social al que está destinada. Así que junto a medidas tecnológicas (cuyos exponentes más conocidos son quizás las energías renovables o las biotecnologías), es necesario adaptar las estructuras sociales, económicas y políticas, y educar a la ciudadanía, en todos los estratos sociales, y a las instituciones para promover la responsabilidad solidaria (usando palabras de Adela Cortina), el cambio en los hábitos y formas de vida, y la creación de una «democracia planetaria» en la que la globalización lo sea no sólo de los productos financieros, sino también de los derechos humanos (que están estrechísimamente vinculados al desarrollo), la justicia social y la política medioambiental.

Aunque se trata de una expresión controvertida, parten del concepto de desarro- llo sostenible o sustentable, como modelo a seguir para guiar el progreso humano. La definición más usual es: «es el desarrollo que satisface las necesidades de esta generación sin comprometer las capacidades de las siguientes generaciones para satisfacer sus propias necesidades». La novedad del concepto descansa en que, cuando se enunció, por primera vez se caía en la cuenta de la limitación del mundo, de que sus recursos son agotables. Así, hace una o dos generaciones empezaba a extenderse la conciencia de que es necesario proteger el planeta. Era el fin del sueño del progreso sin fin. Dando un paso más, los autores defienden la posibilidad de un «desarrollo sin crecimiento», entendiendo el desarrollo como el aumento de potencialidades, sin necesidad de incrementar cuantitativamente la producción o el consumo de recursos energéticos, por ejemplo. También defienden el concepto de sostenibilidad social, en el que se da tanto una solidaridad intergeneracional como intrageneracional.

La cuestión de los desequilibrios Norte-Sur, o centro-periferia, está muy presente también en la obra de Vilches y Gil. De hecho varios capítulos están dedicados a analizar la problemática de la sostenibilidad desde el punto de vista del subdesarrollo, y las consecuencias de estos desequilibrios sobre el medio ambiente y la generación de violencia y conflictos. Por ejemplo, la pobreza conduce, en la búsqueda rápida de soluciones, a la sobreexplotación del medio ambiente $\mathrm{y}$, por tanto, a su degradación.

Avanzar más allá del concepto de desarrollo sostenible es también uno de los intereses principales de Navegando contra el viento. Sus autores, Rodrigo Arocena y Judith Sutz, apuestan por un «desarrollo humano autosustentable», en el que, siguiendo al Premio Nobel Amartya Sen, el desarrollo se entienda como libertad, como desarrollo humano, y que consiste en ampliar las posibilidades y potencialidades de los hombres, y, por lo tanto, disminuir 
la desigualdad. La tensión entre innovación y desarrollo, y, en concreto, ofrecer una reflexión sobre la innovación tecnocientífica desde la perspectiva de los países que están lejos de sus centros tradicionales es el tema de esta obra escrita por dos expertos uruguayos en la materia. Además, la obra constituye también una atractiva introducción a la teoría de la innovación tecnocientífica para aquellos que deseen iniciarse en él, con el atractivo del enfoque «desde la periferia» y el compromiso social.

En una primera parte, el libro de Arocena y Sutz ofrece un panorama de los procesos sociales que constituyen la denominada innovación técnico-productiva. Los autores mantienen, siguiendo la línea de los estudios CTS, un concepto de innovación no sólo técnico, sino social: no sólo se trata de analizar las novedades científico-técnicas, sino también, y de forma primordial, de analizar su implantación en un contexto de práctica determinado. De este modo la innovación es concebida no como un evento aislado, sino como un proceso que combina diversas dinámicas sociales, en las que interactúan actores heterogéneos. En este afán por ofrecer una perspectiva social y sistémica de la innovación, siguiendo los pasos de las teorías elaboradas por autores como Freeman o Nelson (Freeman, C., 1982; Nelson, R. R. y Nelson, S. G, 1982), realizan un minucioso recorrido por todos los actores implicados en este proceso, desde los receptores de la innovación, trabajadores y consumidores más o menos pasivos, hasta las instituciones públicas o privadas (universidad, empresas, laboratorios de I + D..., gobiernos que diseñan políticas de apoyo a la innovación...), y por las formas en que estos actores pueden y deben estar implicados en el proceso para hacer posible la innovación científico-tecnológica en su contexto. $\mathrm{Su}$ concepción defiende una ligazón fundamental entre innovación y cultura, con influencias abundantes y recí- procas entre ambas, como la que ya en 1934 trazaba Lewis Mumford en su obra Técnica y civilización. La tecnología no evoluciona en un proceso lineal y unidireccional, sino que avanza siguiendo un camino en el que se van abriendo múltiples opciones, cada una de las cuales responde a intereses sociales, valores, contextos culturales... la tecnología es, siguiendo a Mumford, un factor de estructuración de prácticas y representaciones del mundo, como refleja el concepto de «imaginario tecnológico». Esta elección de opciones tecnológicas puede darse de forma cooperativa entre diferentes actores, o ser un desencadenante de conflicto social.

La perspectiva histórica está siempre presente en este trabajo, y también, como en la obra de Vilches y Gil, la comparación Norte-Sur o, como prefieren decir los autores, centro-periferia. Analizan cómo los caminos históricos de la innovación siempre han funcionado de manera «natural» en el Norte, donde existía la capacidad para producir y recibir innovaciones tecnológicas, y de manera sobreimpuesta en el Sur, donde se pretendió hacer entrar la innovación por medio de importaciones irreflexivas y fuera de contexto de productos tecnológicos, pero sin crear el tejido social necesario para asumir la innovación y promoverla endógenamente. De acuerdo con esta visión periférica, dedican toda la segunda parte de la obra a analizar la relación entre innovación y subdesarrollo, y cómo la ciencia y la tecnología están implicadas con la desigualdad entre regiones. Su estudio histórico sigue los pasos de la obra de Freeman sobre cómo se ha configurado el sistema mundial de centroperiferia desde la Revolución Industrial, cuando comienza la expansión industrial de los países del Norte y la situación de dependencia del Sur. Esta dependencia es la que provoca que los países periféricos no sólo sean atrasados (en el sentido en que deben recorrer el camino que antes 
transitaran los países centrales), sino también subdesarrollados, en una situación en la que la posición superior de unos determina la posición interior de los otros, en un círculo vicioso que se perpetúa a sí mismo.

Además, la sociedad contemporánea se caracteriza por haber dejado de ser una sociedad industrial, y haber pasado a estar centralizada en torno al conocimiento, que en los últimos tiempos ha adquirido una apabullante importancia económica: si la Revolución Industrial modificó profundamente la sociedad, cambiando de forma radical la forma de vida de las personas y su forma de relacionarse, una nueva revolución tecnológica ha vuelto a conmocionar el panorama a finales del siglo XX. Ésta es la revolución protagonizada por las ciencias de la comunicación, la electrónica y las telecomunicaciones, que ha desembocado en profundos cambios en el mundo del trabajo, en la estructura de las empresas y las instituciones, y, en general, en las relaciones entre Ciencia, Tecnología y Sociedad. La sociedad resultante es una «sociedad económica basada en el conocimiento y monitorizada por la innovación», en la que además el conocimiento es un requisito básico para esta última, y por lo tanto la distribución del progreso científico-técnico corre en paralelo con la distribución del conocimiento. La inversión en ciencia y tecnología en el Norte es 19 veces mayor que en el Sur. En el Norte los sistemas de producción industrial están articulados con los sistemas de producción de conocimiento, y además este último se entiende como un recurso nacional y propio, con una importancia estratégica. En el Sur, en cambio, no se valora el conocimiento propio, sino que se importa cuando se necesita, de forma no conectada con el sistema productivo. Esta diferencia en la valoración del conocimiento es consecuencia de lo que denominan Arocena y Sutz «divisorias del aprendizaje»: las diferencias en índice de alfabetización y acce- so a estudios terciarios, entre otras, muestran una diferente distribución del conocimiento según la cual en el Norte es posible desarrollar mecanismos de innovación que demandan continuamente la mejora de las habilidades existentes y del conocimiento, mientras que en el Sur este desarrollo no es posible. La desigual distribución del conocimiento es entonces la clave principal de la diferencia Norte-Sur y entre grupos sociales. Por esto la transición hacia una nueva sociedad del conocimiento en el Norte no hace sino agrandar la brecha con el Sur, que una vez más vuelve a quedarse atrás. Las diferencias en el conocimiento son las más importantes, porque afectan directamente a la capacidad para mejorar las propias situaciones de desventaja. En conclusión, «el conocimiento es el más poderoso agente de prosperidad y desigualdad».

A propósito de esta cuestión, la obra de Jacques Marcovitch La universidad (im)posible, también editada en la colección, puede ofrecer una ilustración interesante: Marcovitch, desde su experiencia como rector de la Universidad de São Paulo en Brasil, plantea los principales retos a los que se enfrenta la universidad pública en un país periférico dentro de la sociedad del conocimiento, en todas sus dimensiones: investigación, enseñanza y extensión. Quizá la obra resulte demasiado localista en comparación con el resto, dado que ciñe su reflexión a una institución muy concreta, la Universidad de São Paulo, y a su perspectiva determinada por la posición de su autor (algo, por otra parte, siempre inevitable en mayor o menor medida, como muy bien destacan los estudios CTS), pero es sin duda una buena aproximación al problema de la generación de conocimiento en el lado sur de esa «divisoria del aprendizaje».

Volviendo a Arocena y Sutz, su obra no deja de lado la problemática del riesgo, inherente cada vez más al nuevo papel del conocimiento, y uno de los principales 
aspectos sobre los que se configuran las conflictivas relaciones que forman parte del mecanismo de la innovación. Como también recordaban Vilches y Gil en Construyamos un futuro sostenible, la Revolución Industrial, y en concreto los desarrollos tecnológicos contemporáneos, han producido problemas ambientales globales. Estos problemas ocasionados por la ciencia y la tecnología moderna se observan de una forma aún más urgente desde el subdesarrollo, porque, como ocurre con el conocimiento, los riesgos tampoco se reparten de forma igualitaria: en el Sur, los problemas tienen una dimensión mayor, y también son cualitativamente diferentes (en parte, porque las necesidades acuciantes hacen que se prime la producción por encima de la seguridad, y en parte porque los países desarrollados «aparcan» fuera de sus fronteras las actividades dañinas o peligrosas, que los países periféricos no pueden permitirse rechazar).

Si bien el objetivo de esta obra no está tan encaminado a la propuesta de soluciones como la obra de Vilches y Gil, sino más bien al estudio y planteamiento de los problemas, los autores sí se animan con unas cuantas propuestas de carácter general. Una de ellas es la necesidad de potenciar en el Sur líneas de investigación orientadas al desarrollo, con miras a largo plazo y carácter interdisciplinar. También creen necesario fomentar la democratización del conocimiento, en sus dos versiones: la comprensión de la ciencia y la tecnología, y la participación de la ciudadanía en la definición de políticas. En definitiva, su propuesta de buscar un desarrollo humano autosustentable pasa por generar endógenamente el desarrollo, y buscar soluciones plurales y colectivas, que permitan potenciar desde dentro, creando las interconexiones que faltan - por ejemplo, redes interactivas de aprendizaje-, los Sistemas Nacionales de Innovación de los países del subdesarrollo, y romper las divisorias del aprendizaje.
En conclusión, esta colección aborda los principales temas de los estudios sociales de la ciencia y la tecnología con una gran riqueza de perspectivas y enfoques. Sus autores proceden tanto del ámbito académico de las humanidades y las ciencias sociales como del de las ciencias naturales o las instituciones públicas. Escriben desde situaciones muy localizadas, o con enfoques intencionadamente globales. Desde el Norte desarrollado o desde el Sur que pretende hacerse un hueco en la sociedad del conocimiento. $\mathrm{Y}$ en todos encontramos temas como el riesgo científico-tecnológico y su evaluación, las consecuencias para la sociedad de la innovación científico-tecnológica, el reparto equitativo de la riqueza y los problemas ambientales y sociales producidos por el desarrollo, o los problemas éticos que suscita la investigación científica, que han sido desde su aparición fundamentales para este campo de estudio.

Quizás lo más interesante de esta colección es que, considerando las obras editadas hasta el momento en su conjunto, nos permite, volviendo a la idea con la que empezábamos este artículo, reflexionar sobre la ciencia y la tecnología en dos frentes diferenciados aunque inseparables: la omnipresente (en ocasiones incluso asfixiante) mediación de la ciencia y la tecnología en todos los aspectos de la vida en el mundo desarrollado, por un lado, y la desesperante ausencia de ellas en el mundo periférico. Los estudios CTS tradicionalmente sólo se habían dedicado (hablando en términos generales) al primero de ellos, eran estudios «desde el Norte». Estudios como los que componen esta colección permiten abrir el campo de sus reflexiones hacia otras latitudes y otras temáticas, y son una muestra de otra de las máximas que rigen la investigación CTS: la multiplicación de perspectivas y actores enriquece los análisis y la comprensión de cualquier objeto de estudio, por ejemplo, las relaciones entre Ciencia, Tecnología y Sociedad. 


\section{BIBLIOGRAFÍA}

Arocena, R., y Sutz, J.: Navegando contra el viento, Ciencia, Tecnología y Subdesarrollo, Madrid, Cambridge University Press/OEI, próxima aparición.

Beck, U.: La sociedad del riesgo, Barcelona, Paidós, 1998 [1986].

Bijker, W.; Hughes, T., y Pinch, T., The Social Construction of Technologycal Systems: New Directions in the Sociology and History of Technology, Cambridge, The MIT Press, 1987.

CARSON, R.: La primavera silenciosa, Barcelona, Grijalbo, 1980 [1962].

ECHEVERRÍA, J.: Introducción a la metodología de la ciencia: la filosofía de la ciencia en el siglo XX, Madrid, Cátedra, 1999.

FREEMAN, C.: The Economics of Industrial Innovation, Londres, Pinter, 1982 [1982].

Funtowicz, S., y Ravetz, J.: La ciencia postnormal, ciencia con la gente, Barcelona, Icaria, 2000 [1993].

GonzÁlez GARCÍA, M., et al.: Ciencia, Tecnología y Sociedad: una introducción al estudio social de la Ciencia y la Tecnología, Madrid, Tecnos, 1996.

Jones, M. V., A Technology Assessment Methodology: Some basic proposi- tions. Washington DC, Office of Science and Technology/The Mitre Corporation, 1971.

López Cerezo, J. A., y Luján, J. L.: Ciencia y política del riesgo, Madrid, Alianza, 2000.

López Cerezo, J. A., y González García, M. I.: Políticas del bosque, Madrid, Cambridge University Press/OEI, 2002.

MARCOVITCH, J.: La Universidad (im)posible, Madrid, Cambridge University Press/OEI, 2002.

MuÑoz, E.: Biotecnología y sociedad, Cambridge University Press/OEI, 2002.

MumFord, L.: Técnica y civilización, Madrid, Alianza, 1987 [1934].

Nelson, R. R., y Nelson, S. G.: An Evolutionary Theory of Economic Change, Cambridge, Harvard U. P., 1982.

RosZAK, T.: El nacimiento de una contracultura, Barcelona, Kairós, 1970, [1968].

SAREWITZ, D.: Frontiers of Illusion: Science, Technology and the Politics of Progress, Philadelphia, Temple University Press, 1996.

VIlches, A., y GIL, D.: Construyamos un futuro sostenible, Madrid, Cambridge University Press/OEI, 2003. 